\title{
Lifespan changes in multi-tasking: Concurrent walking and memory search in children, young, and older adults
}

\author{
Ralf Th. Krampe ${ }^{\mathrm{a}, *}$, Sabine Schaefer ${ }^{\mathrm{b}}$, Ulman Lindenberger ${ }^{\mathrm{b}}$, Paul B. Baltes ${ }^{\mathrm{b}}$ \\ a Department of Psychology, University of Leuven (KU Leuven), Tiensestraat 102, bus 3715, B-3000 Leuven, Belgium \\ ${ }^{\mathrm{b}}$ Max-Planck Institute for Human Development, Berlin, Germany
}

\section{A R T I C L E I N F O}

\section{Article history:}

Received 9 February 2010

Received in revised form 10 October 2010

Accepted 11 December 2010

\section{Keywords:}

Dual-task

Lifespan

Semantic fluency

Cognitive resources

\begin{abstract}
A B S T R A C T
We investigated dual-task performance of cognitive (semantic fluency) and sensorimotor tasks (walking) in 120 children and adults from four age groups (9-year olds, $M=9.52$ years; 11 -year olds, $M=11.51$ years; young adults, $M=25.34$ years; older adults, $M=64.28$ years; $N=30$ per group). Distances walked during $90 \mathrm{~s}$ and numbers of category exemplars generated in the semantic fluency task showed an inverted U-shape function with age. In line with general resource models proportional dualtask costs in walking also showed a U-shaped relation as a function of age with pronounced decrements in the youngest and oldest groups. Only 9-year olds showed significant costs in the cognitive task. Individual differences in single-task performance accounted for more than half of the variance in dualtask performance. Reliable age-related residual variance implicated additional factors particularly in children's developing multi-tasking performances.
\end{abstract}

(c) 2010 Elsevier B.V. All rights reserved.

\section{Introduction}

Engaging in concurrent sensorimotor and cognitive activities like recalling agendas for upcoming meetings while walking towards our destinations is daily-life routines, which young and middle-aged adults typically consider as timesaving rather than as challenging. Older adults, in contrast, experience pronounced performance decrements (dual-task costs), when engaging in two activities at the same time, even if one task requires seemingly automatic skills like walking or maintaining postural stability $[1,2]$. Interestingly, older adults tend to prioritize balancing or walking over cognitive performance (i.e., accrue higher dual-task costs in the latter), at least when the sensorimotor task provides real challenge [3-7]. Studies investigating multi-tasking in children point to improvements throughout middle-childhood [for reviews, 8]. Few studies investigated combinations of cognitive and sensorimotor tasks in children, however, similar prioritization of balance over concurrent cognitive tasks was

\footnotetext{
This research was conducted in the context of the project Interplay of Cognitive and Sensorimotor Functions (co-directed by Ralf Th. Krampe and Paul B. Baltes). Unfortunately Paul Baltes died in 2006 when this work that he inspired and initiated was well under way. The authors like to thank Gabriele Faust, Nina Blaskewitz, Stefanie Dabrowski, Anna Gronostaj, Simone Ränker, Sarah Risse, and Gabi Schmid for their help with data collection and Werner Scholtysik and Bernd Wischnewski for technical support and programming. Support through BOF grant OT25/05 from the Research Council of KU Leuven and FWO-grant (Fonds Wetenschappelijke Onderzoek) G.0379.06 to the first author is acknowledged.

* Corresponding author. Tel.: +32 16 326104; fax: +32 16326144.

E-mail address: Ralf.Krampe@psy.kuleuven.be (R.Th. Krampe).
}

observed in 9-11 year old children [9]. To our knowledge, no study has as yet compared concurrent cognitive and sensorimotor performances in children and older adults within the same paradigm.

Two basic accounts of dual-task costs and related agedifferences have been discussed, namely, first, task competition for general processing resources along with resulting shortages thereof [10] and second, limitations in task-coordination strategies or resource allocation [11]. Prominent versions of general-resource accounts attribute age-differences to the development of processing speed with gains throughout childhood being followed by stability in young and middle adulthood and, finally adult-age related declines [12,13]. Taken together these findings suggest an inverted U-shaped relation between age and available resources across the lifespan. The key assumption of general-resource models is that the same individual differences that constrain single-task performance determine dual-task performance. The critical test for this account is to evaluate age-differences in dualtask performance after individual differences in single-task performance are statistically controlled for (i.e., by means of hierarchical regression analyses). Two studies [14,15] indeed found that larger dual-task costs in older compared with young adults were greatly diminished or disappeared altogether when single-task performance was controlled for.

Accounts focusing on flexibility of resource-allocation and taskcoordination do not preclude general resources as a source of dualtask effects. Instead they posit that the ability to prioritize one task over another or to strategically organize and schedule concurrent responses add to dual-task costs over and above general resource 
demands. For example, Irwin-Chase and Burns [16] showed that after individual differences in children's capacity for single-task performance were controlled for, age differences between 8- and 11-year old children only existed, when tasks had different priorities. In older adults, Kramer et al. demonstrated similar abilities in implementing differential priority schemes as in young adults [17]. Young children suffer pronounced dual-task decrements if one of the tasks involves a strategic component, like organizing their retrieval efforts in cumulative recall tasks [18-20]. Strategic differences between young and older adults in coordinating concurrent task demands have been demonstrated by Glass et al. [21] in their study of the psychological-refractory period.

The present study had three goals. First, we assessed the Ushape prediction for dual-task costs derived from the general resource model. Second, we asked whether children and adults differ with respect to prioritizing concurrent cognitive and sensorimotor tasks, when no imminent threat to postural stability was involved. Third, we evaluated the explanatory scope of the general resource model of age-differences in dual-task costs by considering correlations between single- and dual-task performances. To this end, we tested children, young, and, older adults with a combination of walking and semantic fluency, a task in which participants generate members of a specific semantic category (e.g., four-legged animals) for a fixed trial duration. Semantic fluency involves strategic scheduling and monitoring [22]. Performance increases linearly in children between the ages of 5 and 12 years [23] and small, but robust decrements were found in elderly compared with young adults [24].

\section{Methods}

\subsection{Participants}

Originally, 138 participants in four age-brackets (9- and 11-year-old children, young and older adults) were recruited from the subject pool of the Max Planck Institute for Human Development and through advertisements at local university campuses, schools, and supermarkets. They received 20 Euro for their participation. Data from nine participants had to be excluded due to equipment malfunction. During an extra session we screened older individuals using easy walking exercises, assessments of postural stability, and extensive questionnaires on their medical history and incidences of falling. Individuals who reported recent falls or a history of severe illnesses like heart attacks and strokes were excluded from the study. This was the case for six older participants. Three 9-year olds with ADHD diagnosis or health problems that prevented participation in school sports were also excluded. The final sample consisted of 30 participants (half female) in each age-bracket. Sample characteristics are shown in Table 1.

\subsection{Word-fluency task}

A recurrent problem in age-comparative dual-task research is that tasks over- or under-challenge individual groups such that ceiling or floor effects in single-task performance render the demonstration of dual-task costs impossible. For these reasons we used different categories for children and adults (Table 2) with six identical items in the two sets. Categories for adults came from Mayr and Kliegl [22]. Categories for children were based on our own calibration study in which 249 -year olds (none of who participated in the main study) performed the fluency task for $90 \mathrm{~s}$ trials on 24 categories. Four raters coded tape-recorded verbalizations (interrater reliability: Cronbach's $\alpha=.988$ ). Based on the number of correct
Table 2

Semantic categories used in the fluency task.

\begin{tabular}{|c|c|c|c|}
\hline \multirow[t]{2}{*}{ Age group } & \multicolumn{3}{|l|}{ Difficulty level } \\
\hline & Easy & Medium & Difficult \\
\hline \multirow[t]{8}{*}{ Children } & Drinks and liquids & School subjects & Diseases \\
\hline & Fruits \& vegetables & Sports & Furniture \\
\hline & $\begin{array}{l}\text { a Four-legged } \\
\text { animals }\end{array}$ & Handcraft material & $\begin{array}{l}\text { Fairytale } \\
\text { characters }\end{array}$ \\
\hline & ${ }^{\mathrm{a} C l o t h e s}$ & Plants, flowers, trees & TV shows \\
\hline & ${ }^{\mathrm{a}}$ Colors & ${ }^{\mathrm{b}}$ Pokemons & Sweets \\
\hline & ${ }^{\mathrm{a} B o d y}$ parts & ${ }^{\mathrm{b}}$ Cities and Countries & ${ }^{\mathrm{a}}$ Vehicles \\
\hline & Female first names & birds & $\begin{array}{l}\text { aMusical } \\
\text { instruments }\end{array}$ \\
\hline & Male first names & ${ }^{\mathrm{b}}$ Professions & Games and toys \\
\hline \multirow[t]{8}{*}{ Adults } & ${ }^{a}$ Body parts & Vegetables & Traffic signs \\
\hline & $\begin{array}{l}\text { aFour-legged } \\
\text { animals }\end{array}$ & Spices & Writing utensils \\
\hline & ${ }^{\mathrm{a}}$ Colors & $\begin{array}{l}\text { Nonalcoholic } \\
\text { beverages }\end{array}$ & $\begin{array}{l}\text { Communication } \\
\text { means }\end{array}$ \\
\hline & ${ }^{\text {avehicles }}$ & Flowers & Insects \\
\hline & Birds & ${ }^{\mathrm{b}}$ Kitchen utensils & Fabrics \\
\hline & ${ }^{\mathrm{a} C l o t h e s}$ & ${ }^{\mathrm{b}}$ Weather phenomena & Sciences \\
\hline & $\begin{array}{l}{ }^{\mathrm{a}} \text { Musical } \\
\text { instruments }\end{array}$ & ${ }^{\mathrm{b}}$ Fish & Fluids \\
\hline & Food items & ${ }^{\mathrm{b}}$ Building materials & Criminal acts \\
\hline
\end{tabular}

a Categories appearing in children- and adults-sets.

b Categories used during warm-up trials.

exemplars we assigned categories to easy $(M=16.0, \mathrm{SD}=2.5$, range 13.7-19.5), medium $(M=11.6, \mathrm{SD}=3.6$, range $11.0-13.4)$, and difficult conditions $(M=9.4$, $\mathrm{SD}=2.3$, range $8.0-10.9$ ).

Four items from the medium difficulty level in each group were used during warm-up trials and not included in the final analyses. Thus, participants performed eight semantic fluency trials each on easy and difficult categories and four on medium categories. Half of the trials in each difficulty level were performed under single-, the other half under dual-task conditions.

\subsection{Procedure}

Participants were tested individually during two 1-h sessions. Standard versions of psychometric tests for children [25] and adults [26] measuring vocabulary knowledge, working memory capacity (Digit-span) and perceptual-motor speed (Digit-symbol substitution) were administered at the beginning of Session 1 and between blocks of experimental trials. Following warm-up trials four blocks of test trials were administered in each session with single-task assessments in fluency and walking tasks at the beginning and ends to balance potential effects of training and transfer among conditions. Participants performed 10 trials in each task and condition (single-task fluency, single-task walking, and dual-task), four in Session 1 and six in Session 2. Fluency categories and order of difficulty levels were counterbalanced across participants and task contexts (i.e., single and dual).

In the walking task participants walked a narrow track (width $19 \mathrm{~cm}$, in curves $19-30 \mathrm{~cm}$ ). Instructions encouraged walking as fast as possible while avoiding to step on the track's outer boundaries. They wore wireless headsets with microphones such that their verbalizations during dual-task trials could be taperecorded. The track was oval (length $24 \mathrm{~m}$ ) with two parallel straight sections (length $9 \mathrm{~m}$ ) connected by half circles of $1.5 \mathrm{~m}$ diameter. Accurate walking and the limited width of the track required participants to set their feet in front of each other with less lateral distance than in normal walking, however different from tandem walking they stepped without feed touching each other. Steps were registered to the nearest millisecond by a Macintosh PowerPC through contacts

Table 1

Sample characteristics. Group means and SDs (in parentheses).

\begin{tabular}{|c|c|c|c|c|}
\hline Variable & 9-Year olds & 11-Year olds & Young adults & Older adults \\
\hline Age (years) & $9.52(.36)$ & $11.51(.29)$ & $24.34(2.21)$ & $64.28(2.42)$ \\
\hline Digit-symbol $^{\mathrm{a}}$ substitution ( $N$ items) & $42.70(7.30)$ & $54.53(7.90)$ & $64.30(9.67)$ & $44.87(8.40)$ \\
\hline Digit-symbol $^{\mathrm{a}}$ substitution (time/item) & $2.89(.46)$ & $2.25(.36)$ & $1.43(.20)$ & $2.08(.42)$ \\
\hline Digit-span forward ${ }^{\mathrm{a}}$ & $7.33(1.30)$ & $8.07(1.53)$ & $8.90(1.92)$ & $7.33(2.06)$ \\
\hline Digit-span backward ${ }^{a}$ & $5.00(1.55)$ & $5.47(1.72)$ & $8.50(1.87)$ & $6.40(1.43)$ \\
\hline Vocabulary $^{\mathrm{a}}$ & $30.41(8.30)$ & $38.55(6.91)$ & $24.92(3.90)$ & $21.80(4.87)$ \\
\hline Height (m) & $1.42(.08)$ & $1.52(.07)$ & $1.75(.09)$ & $1.71(.07)$ \\
\hline Weight (kg) & $35.69(7.07)$ & $45.03(6.37)$ & $70.58(14.69)$ & $70.53(11.62)$ \\
\hline Body mass index & $17.07(2.43)$ & $18.31(1.86)$ & $22.18(3.14)$ & $23.95(3.21)$ \\
\hline
\end{tabular}

a Subtests of the German adaptations of the Wechsler Intelligence Scales for children [25] and adults [26], respectively. 
between the conducive materials under participants' shoes and on the track surface. In the word fluency task participants were instructed to produce as many correct exemplars as possible for one of 24 semantic categories. During single-task trials participants sat next to the experimenter. In dual task trials the experimenter shouted the target category to participants immediately after they started walking. Trial durations were always $90 \mathrm{~s}$

\subsection{Data analysis}

Tape-recorded verbalizations of semantic fluency trials were coded by the same raters as in the pilot study for the number of correct category exemplars. The total distance covered during $90 \mathrm{~s}$ trial durations served as a measure of walking performance. Test-retest reliabilities for both dependent measures were acceptable (Cronbach's $\alpha>.83$, at the level of single age groups). Performance measures were averaged across 10 trials each in single- and dual-task conditions. We conducted mixed-design ANOVAs using age group (4) as between, and task context (single vs. dual) and difficulty of the semantic category in the fluency task (easy, medium, difficulty) as repeated measure factors. Post hoc comparisons were conducted as $t$ tests. Alpha-levels in all tests were set at .05. To allow comparisons between modalities (walking and cognition) and groups differing in single-task baseline, we calculated proportional dual-task costs (absolute costs expressed in percent singletask performance). The conventional criterion of two-SE difference from zero (corresponding to two-tailed significance testing) was used to determine reliable costs. All statistical analyses were conducted with SPSSX (version 13) for the Macintosh.

\section{Results}

\subsection{Semantic fluency}

The mixed-design ANOVA conducted on the numbers of exemplars retrieved in the semantic fluency task showed that simultaneous walking reduced memory performance $(M=15.89$, $\mathrm{SD}=5.40)$ compared with single-task conditions $(M=16.55$, $\mathrm{SD}=5.29), F(1,116)=6.76, M S E=11.45, p<.05$, and this effect was similar across age groups $(p>.75)$, and levels of semantic category difficulty $(p>.50)$. Our manipulations of category difficulty acted as predicted, $F(2,232)=539.82, \quad M S E=8.13$, $p<.001$, and differences between levels were robust at the level of individual age groups. The main effect of age group, $F(3$, $116)=33.43, \quad M S E=88.00, \quad p<.001$, reflected the significant differences between all groups with young adults $>$ older adults $>11$-year olds $>9$ year olds (Fig. 1, top panel). Absolute differences between age groups tended to be larger for easier than for medium or difficult categories, $F(6,232)=11.04, p<.05$, presumable reflecting adults' extended vocabularies. The pattern of results was replicated when we restricted analyses to the six semantic categories administered to all age groups.

\subsection{Walking}

Participants produced a higher percentage of missteps under dual- $(2.67 \%, \mathrm{SD}=3.89)$ compared with single-task conditions $(2.25 \%, \mathrm{SD}=3.19), F(1,116)<4.41, p<.05$, and age groups were similar in this respect. A mixed-design ANOVA on distances covered in four task-context conditions (single task, dual-easy, dual-medium, and dual-difficult categories) produced a main effect of age group, $F(3,116)=21.25, M S E=1540.01, p<.001$, and a main effect of task context, $F(3,348)=169.10, M S E=27.06$, $p<.001$. Effects of task context were similar across age groups $(p>.4)$. Post hoc tests revealed that participants walked further in single- compared with any of the three dual-task conditions, and this effect generalized across age groups (all $p$ 's $<.001$ ). Difficult categories lead to a larger reduction $(M=13.23 \mathrm{~m}, \mathrm{SD}=9.28)$ in walking distances compared with easy $(\Delta M=.73 \mathrm{~m}, \mathrm{SD}=2.58)$ and medium categories $(\Delta M=2.38 \mathrm{~m}, \quad \mathrm{SD}=5.78), \quad t^{\prime} \mathrm{s}(119)>3$, $p$ 's $<.002$. Note, however, distances walked with medium items were longer than for easy categories. Overall differences between age groups followed the pattern young adults $>11$-year olds $>9$ year olds $\approx$ older adults (Fig. 1 , bottom) with significant differ-
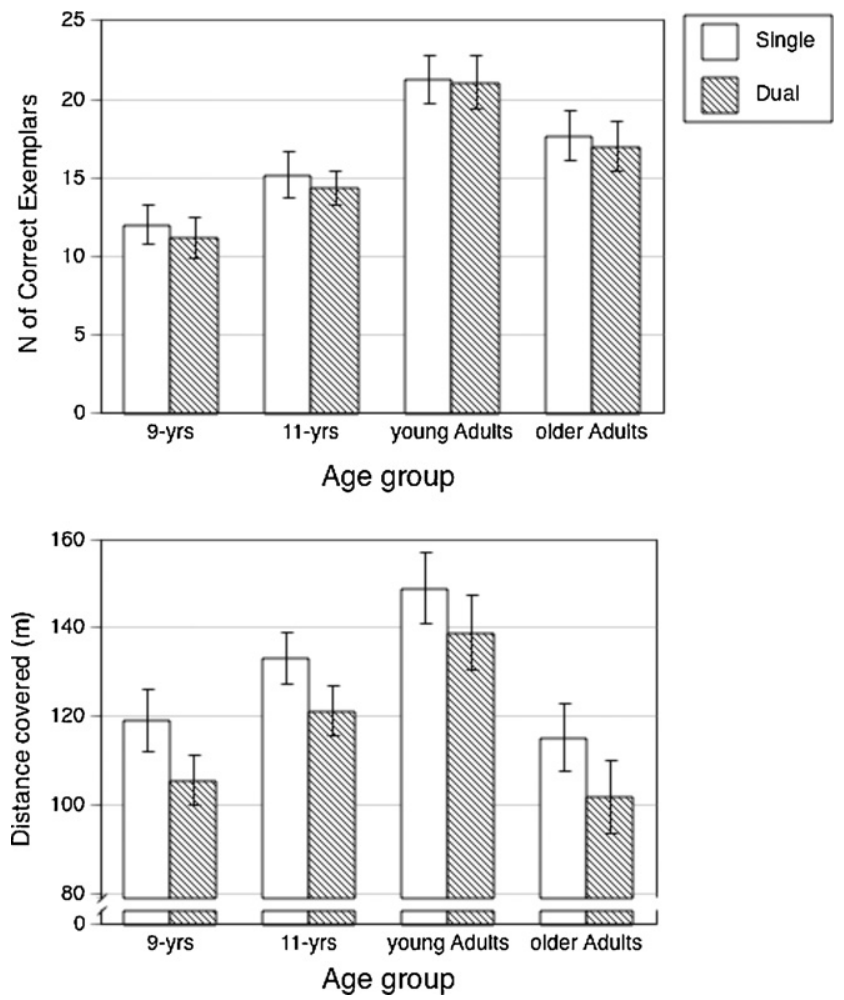

Fig. 1. Mean number of category exemplars retrieved in semantic fluency task (top) and average distance covered in the walking task (bottom) as a function of age group and task context. Error bars represent 95\% between-group confidence intervals.

ences between individual groups, $t$ 's $(58)>3.3, p<.001$, with the exception of 9 -year olds and older adults.

\subsection{Proportional dual-task costs}

Given that effects of category difficulty in the semantic fluency task were similar in single- and dual-task contexts we averaged performance across all items for our calculation of proportional dual-task costs (the same pattern of findings emerges when category difficulty is included). For the walking task, all age groups showed significant proportional costs (marked by asterisks in Fig. 2). An ANOVA on proportional walking costs showed a main effect of age group, $F(3,116)=3.03, M S E=49.04, p<.05$, reflecting the expected U-shape pattern: young adults' costs $(M=7.28 \%$, $\mathrm{SD}=5.68$ ) were smaller than, both, costs in older adults $(M=12.33 \%, \mathrm{SD}=9.65), t(58)=2.47, p<.016$, and costs in 9 -year olds $(M=11.15 \%, \mathrm{SD}=6.58), t(58)=2.44, p<.018$. In between, $11-$

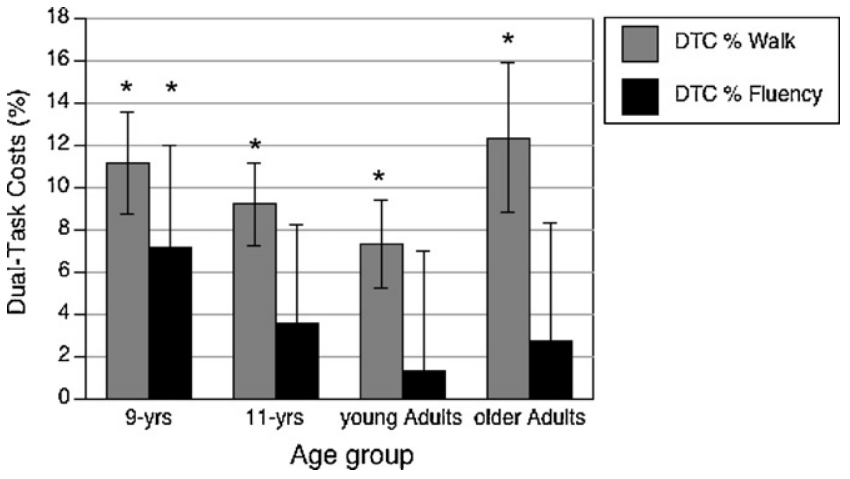

Fig. 2. Proportional dual-task costs in walking and fluency for four age groups. Asterisks mark costs reliably different from 0 by a 2 SE criterion. Error bars represent $95 \%$ between-group confidence intervals. 


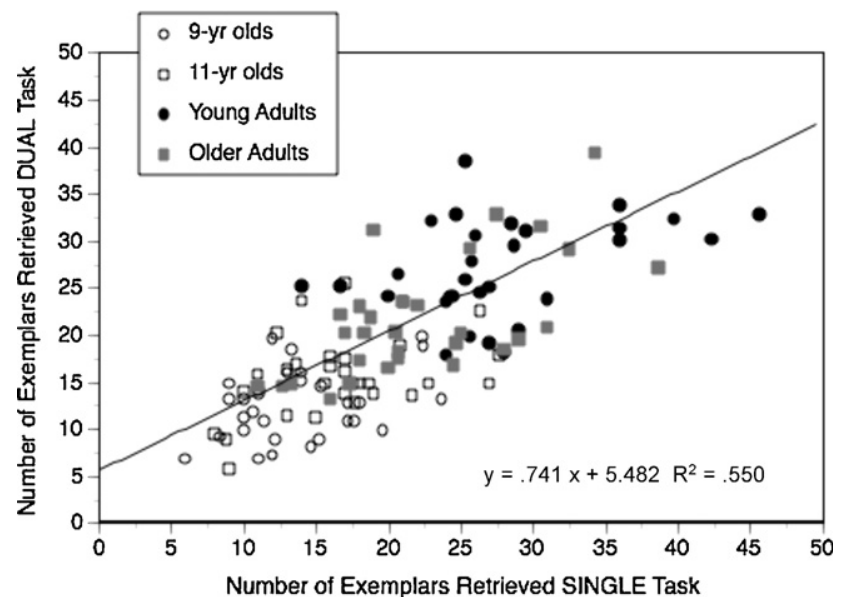

Fig. 3. Linear regression of semantic fluency dual- on single-task performance based on data from all participants $(N=120)$. Performance measures are based on semantic category items that were administered in all age groups.

year olds did not differ significantly from young adults or the two other groups. Only 9-year olds produced significant costs in the fluency task.

To assess group differences in task prioritization, we conducted a mixed-design ANOVA on proportional dual-task costs with age group (4) as between- and task modality (walking vs. fluency) as within-subject factors. The only significant effect related to task modality, $F(1,116)=19.74, M S E=121.44, p<.001$, reflecting higher costs in walking $(M=9.98 \%, \mathrm{SD}=7.18)$ compared with fluency $(M=3.66 \%, \mathrm{SD}=14.28)$. This outcome suggests similar resource allocation preferences in different age groups.

\subsection{Correlations between single- and dual-task performances}

Support for an extension of the general resource model would be gained by showing that the same relation between single- and dual-task performance holds for groups from different phases of the lifespan. We put this proposition to a test by conducting a linear regression of dual- on single-task fluency performance (Fig. 3) based on those semantic categories (compare Table 2) used for all groups. We considered this analysis conservative with respect to potential group differences in terms of range of item difficulties. Model fit was significant, $F(1,118)=143.93$, $M S E=25.31, \quad p<.001$, and variance accounted for large, $R^{2}=.550$. However, residuals were not equally distributed across age groups, but significantly more positive in young adults $(M=2.49, \mathrm{SD}=5.56)$ compared with 9 -year olds $(M=-2.22$, $\mathrm{SD}=3.92)$ and 11 -year olds $(M=-1.15, \mathrm{SD}=4.36), t^{\prime} \mathrm{s}(58)>2.8$, $p<.005$. This also held for the comparison of older adults $(M=.87$, $\mathrm{SD}=4.90$ ) and 9-year olds, $t(58)=2.69, p<.01$. As a result, age group significantly increased the variance accounted for when added to the regression model in a second step, F-change (1, $117)=15.19, p<.001, \Delta R^{2}=.052$. The bottom line was that children's actual performance was worse than predicted on the basis of their single-task performance, whereas the same model underestimated young adults' performance.

\section{Discussion}

Our study is the first to investigate lifespan development in multi-tasking for a combination of sensorimotor (walking) and cognitive (semantic fluency) tasks within the same experimental paradigm. In line with the predictions of the general resource model, we found a U-shaped relation in proportional dual-task costs for walking with significant differences emerging between young adults on the one hand and 9-year old children and older adults, on the other. Only 9-year olds showed significant costs in the semantic fluency task, a finding resonating with earlier reports about this age group's disadvantages in tasks involving strategic components like organizing retrieval efforts in cumulative recall [18-20]. Dual-task costs in walking were higher for more difficult semantic categories in the concurrent fluency task indicating that cognitive processes rather than interference from simultaneous motor output (i.e., verbalization) must be seen as the source of dual-task costs.

While previous studies reported prioritization of walking or postural control over cognitive tasks in children [9] or older adults [6], participants in our study, irrespective of their ages, showed higher dual-task costs in walking than in semantic fluency tasks. Like these authors proposed, we consider the critical factor in prioritization the perceived difficulty of the sensorimotor task. Participants in the aforementioned studies had to balance on ankle-disc boards [9] or walk a parcours with obstacles [6]. In our study, participants walked on flat ground and their postural stability was never really threatened. Taken together with these earlier findings, our results highlight older adults' and children's abilities to accommodate their resource allocation in multi-tasking to the ecological constraints of the setting $[3,5,7]$.

The general resource model maintains that the same individual differences in cognitive resources that constrain single-task performance determine dual-task performance. In its most radical form this implies identical relations (i.e., one regression model) for groups from different phases of the lifespan. When we tried to extend this approach to children's dual-task performances, however, limitations became apparent. In particular, differences between children and young adults were not sufficiently captured by the parsimonious general resource model. Taken together with the additional dual-task costs in fluency we observed in 9-year olds only, it is tempting to hypothesize (strategic) task-coordination deficits in childhood over and above what is captured by singletask performance. However, our findings can only point the direction in this respect, because we agree with those authors who have argued that conclusions about such mediator relations (i.e., general resources mediating age-differences in dual-tasking) cannot be drawn from hierarchical regression analyses and related techniques $[27,28]$. From this perspective, correlations between single- and dual-task performance can be used to show the limitations of extant accounts, but otherwise only be a first step towards understanding component processes.

In conclusion, we demonstrated that high-level cognitive processes interfere with seemingly automatic sensorimotor processes like walking. The mutual costs accrued by such interference are pronounced at opposite ends of the lifespan. Our approach of testing children and older adults within the same dual-task paradigm holds promise for future research, which should extend the search for age-related differences towards multi-tasking strategies like differential emphasis or response scheduling.

\section{Conflict of interest statement}

The authors declare they have no financial or personal relationships with other people or organizations that could inappropriately influence their work.

\section{References}

[1] Lindenberger U, Marsiske M, Baltes PB. Memorizing while walking: increase in dual-task costs from young adulthood to old age. Psychology and Aging 2000;15:417-36.

[2] Woollacott M, Shumway-Cook A. Attention and the control of posture and gait: a review of an emerging area of research. Gait and Posture 2002;16:1-14. 
[3] Brown LA, Sleik RJ, Polych MA, Gage WH. Is the prioritization of postural control altered in conditions of postural threat in younger and older adults? Journal of Gerontology Medical Sciences 2002;57A:M785-92.

[4] Doumas M, Rapp M, Krampe RT. Working memory and postural control: adultage differences in potential for improvement, task-priority and dual-tasking. Journal of Gerontology Series B Psychological Sciences and Social Sciences 2009;64:193-201.

[5] Doumas M, Smolders C, Krampe RT. Task prioritization in aging: effects of sensory information on concurrent posture and memory performance. Experimental Brain Research 2008;187:275-81.

[6] Li KZH, Lindenberger U, Freund AM, Baltes PB. Walking while memorizing: age-related differences in compensatory behavior. Psychological Science 2001;12:230-7.

[7] Rapp M, Krampe RT, Baltes PB. Adaptive task prioritization in aging: selective resource allocation to postural control is preserved in Alzheimer disease. American Journal of Geriatric Psychiatry 2006;14(1):52-61.

[8] Guttentag RE. Age differences in dual-task performance: procedures, assumptions, and results. Developmental Review 1989;9(2):146-70.

[9] Schaefer S, Krampe RT, Lindenberger U, Baltes PB. Age differences between children and young adults in the dynamics of dual-task prioritization: body (balance) vs. mind (memory). Developmental Psychology 2008;44:747-57.

[10] Crossley M, Hiscock M. Age-related differences in concurrent-task performance of normal adults - evidence for a decline in processing resources. Psychology and Aging 1992;7(4):499-506.

[11] Baddeley AD, Della Sala S, Papgno C, Spinnler H. Dual-task performance in dysexecutive and nondysexecutive persons wih frontal lesions. Neuropsychology 1997;11:187-94.

[12] Cerella J. Information processing rates in the elderly. Psychological Bulletin 1985;98:67-83.

[13] Kail R, Salthouse TA. Processing speed as a mental capacity. Acta Psychologica 1994;86:199-225.

[14] Ribaupierre AD, Ludwig C. Age differences in divided attention: is there a general deficit? Experimental Aging Research 2003;29(1):79-105.

[15] Verhaeghen P, Steitz DW, Sliwinski MJ, Cerella J. Aging and dual-task performance: a meta-analysis. Psychology and Aging 2003;18(3):443-60.
[16] Irwin-Chase H, Burns B. Developmental changes in children's abilities to share and allocate attention in a dual task. Journal of Experimental Child Psychology 2000;77(1):61-85.

[17] Kramer AF, Larish JF, Weber TA, Bardell L. Training for executive control: task coordination strategies and aging. In: Gopher D, Koriat A, editors. Attention and performance XVII. Cognitive regulation of performance: interaction of theory and application. Cambridge, MA: The MIT Press; 1999. p. 617-52.

[18] Bjorklund DF, Harnishfeger KK. Developmental differences in the mental effort requirements for the use of an organizational strategy in free recall. Journal of Experimental Child Psychology 1987;44(1):109-25.

[19] Guttentag RE. The mental effort requirement of cumulative rehearsal: a developmental study. Journal of Experimental Child Psychology 1984;37(1):92-106.

[20] Kee DW, Davies L. Mental effort and elaboration: a developmental analysis. Contemporary Educational Psychology 1988;13(3):221-8.

[21] Glass JM, Schumacher EH, Lauber EJ, Zurbriggen EL, Gmeindl L, Kieras DE, et al. Aging and the psychological refractory period: task-coordination strategies in young and old adults. Psychology and Aging 2000;15(4):571-95.

[22] Mayr U, Kliegl R. Complex semantic processing in old age: does it stay or does it go? Psychology and Aging 2000;15:29-43.

[23] Riva D, Nichelli F, Devoti M. Developmental aspects of verbal fluency and confrontation naming in children. Brain and Language 2000;71:267-84.

[24] Obler LK, Albert ML. Language skills across adulthood. In: Birren JE, Schaie KW, editors. Handbook of the psychology of aging. New York: Van Nostrand Reinhold; 1985. p. 463-73.

[25] Tewes U, Rossmann P, Schallberger U, editors. Hamburg-Wechsler Intelligenztest fuer Kinder (3. Auflage). Hamburg-Wechsler Intelligence Test for Children3rd ed., Bern, Switzerland: Huber; 1999.

[26] Tewes U, editor. Hamburg-Wechsler Intelligenztest fuer Erwachsene (Revision 1991)Hamburg-Wechsler Intelligence Test for Adults (Revised Edition 1991)Bern, Switzerland: Huber; 1991.

[27] Lindenberger U, Pötter U. The complex nature of unique and shared effects in hierarchical linear regression: implications for developmental psychology. Psychological Methods 1998;3:218-30.

[28] Sliwinski M, Hofer S. How strong is the evidence for mediational hypotheses of age-related memory loss? Gerontology 1999;45(6):351-4. 